\title{
Equilibrium Contact Probabilities in Dense Plasmas
}

\author{
B. Militzer \\ Geophysical Laboratory, Carnegie Institution of Washington, \\ 5251 Broad Branch Road, NW, Washington, DC 20015 \\ E.L. Pollock \\ Lawrence Livermore National Laboratory, University of California, Livermore, CA 94550
}

(Dated: July 3, 2018)

\begin{abstract}
Nuclear reaction rates in plasmas depend on the overlap (contact) probability of the reacting ions. Path integral Monte Carlo (PIMC) calculations are used here to determine these contact probabilities, $g(0)$, for the one component plasma (OCP) with emphasis on many-body quantum effects which can lead to order of magnitude changes. An intuitive explanation for these effects is presented. The small $r$ behavior of $g(r)$ for quantum systems and the relation to free energies is then derived and compared to the path integral results. Going beyond the uniform background approximation, electron screening effects and the limits of the "constant energy shift" approximation are discussed. Thermodynamic properties for the quantum OCP are analyzed in a final section.
\end{abstract}

\section{INTRODUCTION}

Due to its importance in calculating nuclear reaction rates, the small $r$ behavior of the radial distribution function, $g(r)$, in ionized systems has been a subject of major interest since the original work of Salpeter ${ }^{1}$. For the uniform background model of a classical plasma, simulation studies have produced a quantitative picture of the role of manybody effects 2.3 . Changes due to quantum effects are less thoroughly understood ${ }^{4.5 .6}$.

This article uses path integral Monte Carlo to compute $g(r)$ and in particular $g(0)$ for dense plasma models. Section II presents extensive results for the quantum one component plasma model of ions in a charge neutralizing background and gives an intuitive explanation for the trends observed. For classical systems $g(0)$ has a simple connection to a free energy difference. The relation is more complicated in the quantum case and section III along with the appendix discusses the small $r$ behavior of $g(r)$ in general. Section IV goes beyond the uniform background approximation to consider the effects of electron screening ${ }^{7}$. The thermodynamic functions from the simulations are given in section $\mathrm{V}$.

The ensuing question of how this information on contact probabilities is ultimately used in a reaction rate calculation $^{8}$ has not been rigorously answered in the literature. The common expression for the equilibrium nuclear reaction rate is obtained by multiplying the experimentally determined cross section for a particular reaction, usually as a function of incident momentum, by a particle flux and averaging over the Maxwellian distribution for the relative incident momentum. Multiplying by the target density gives the reaction rate per volume between species 1 and 2 at temperature $k_{B} T=1 / \beta$

$$
\mathcal{R}_{12}=n_{1} n_{2} \int \sigma(p)\left(\frac{p}{\mu}\right)\left(\frac{\beta}{2 \pi \mu}\right)^{3 / 2} e^{-\beta p^{2} / 2 \mu} d^{3} p .
$$

For charged particle reactions, the cross section $\sigma(p)$ is usually written as a "nuclear" cross section or astrophysical factor times a term representing the Coulomb barrier penetration. This last step has the advantage of permitting cross sections measured at accessible experimental energies to be more reliably extrapolated down to the lower energies relevant to most astrophysical applications.

Different prescriptions have been advanced for modifying this reaction rate to take into account many-body ion and electron screening effects. The most common prescription is to simply multiply the above reaction rate by the relative change in the contact probability, $e^{H(0)}$ defined below. For electron screening effects a constant energy shift (see section IV) in the Coulomb barrier penetration term is, however, frequently used. The work of Brown and Sawyer ${ }^{9}$ perhaps provides a starting point for a fuller discussion of this key question.

\section{OCP CONTACT PROBABILITIES}

Dense plasma effects on nuclear reaction rates are usually described in terms of the enhancement in the contact probability $g(0) . g(0)$ is commonly factored into the two-body term, $g_{b i n}(0)$, and a term, $H(0)$, representing manybody effects,

$$
g(r)=g_{b i n}(r) e^{H(r)}
$$



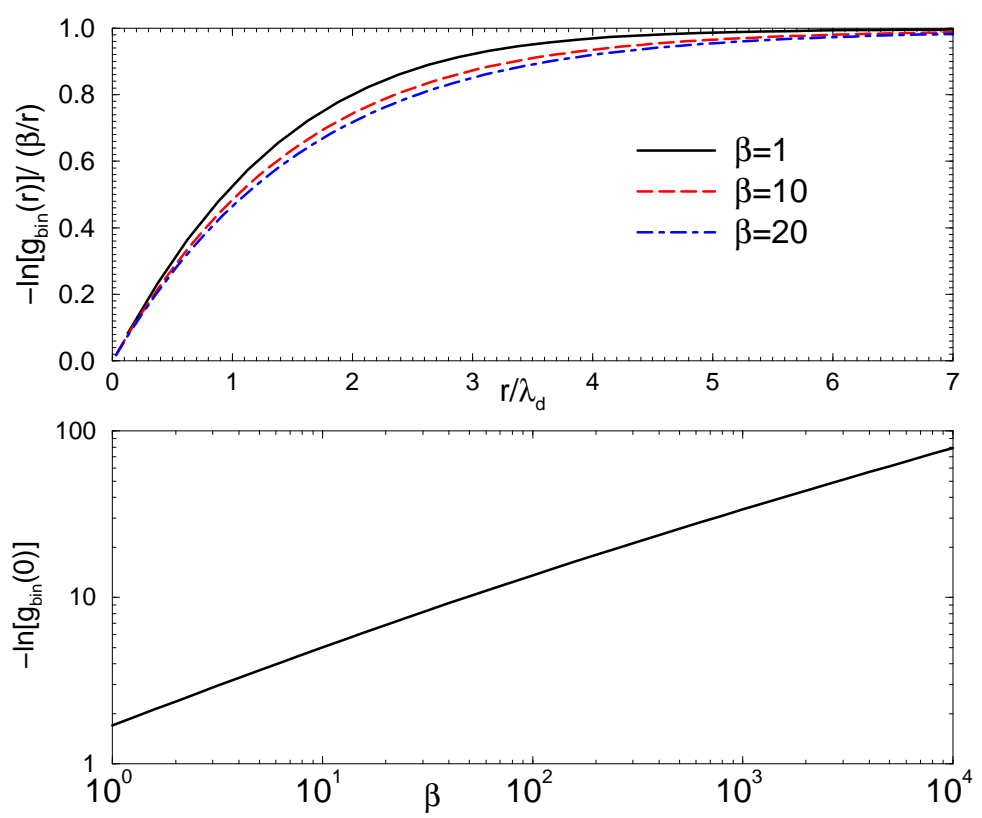

FIG. 1: $g_{b i n}(r)$ for the Coulomb potential. The upper panel shows the convergence to the classical limit for $r$ several times the de Broglie thermal wavelength, $\lambda_{d}$. The lower panel shows the dependence of $g(0)$ on the temperature. The quantum $g(0)$ is finite, unlike the classical limit.

For a classical system $g_{b i n}(r)$ is just $e^{-\beta Z_{1} Z_{2} e^{2} / r}$. For a quantum system $g_{b i n}(r)$ is obtained from the solution of the Bloch equation for the density matrix.

For the repulsive Coulomb potential $g_{b i n}(0)$ has an analytic expression ${ }^{10}$

$$
g_{b i n}(0)=(4 \pi \beta)^{3 / 2} \frac{Z^{3}}{2 \pi} \int_{0}^{\infty} \frac{k e^{-\beta Z^{2} k^{2}} d k}{e^{\pi / k}-1} .
$$

A plot of $g_{b i n}(0)$ is shown in the lower panel of Fig. 1. Unlike the classical $g_{b i n}(r)$ which is zero at the origin, the quantum $g_{b i n}(0)$ is finite at the origin and increases with temperature. As expected $g_{b i n}(r)$ converges to the classical result for $r$ larger than the de Broglie thermal wavelength, $\lambda_{d}^{2}=\hbar^{2} / 2 \pi m k_{B} T$. This is demonstrated in the upper panel of Fig. 1.

In this section, contact probabilities are discussed for the OCP model consisting of a single ion species in a uniform, charge neutralizing background. In the classical limit, the nonideal properties of this model depend only on the dimensionless combination of temperature and density given by the coupling constant $\Gamma=Z^{2} e^{2} / k_{B} T a$, where $a$ is the ion sphere radius defined by $4 \pi a^{3} N / V=1$. $\Gamma$ is a measure of the relative importance of potential to kinetic energy. Contact probabilities for this classical model have been studied starting from the Monte Carlo simulations of Brush, Sahlin and Teller ${ }^{2.11}$. Empirically $H(0)$ is dominated by a linear dependence on $\Gamma$ so it is convenient to define an enhancement factor, $h(0) \equiv H(0) / \Gamma$.

When quantum effects are included both density and temperature must be specified and it is necessary to introduce a quantum parameter $\eta \equiv \Gamma / r_{s}$, with $r_{s}=a / a_{0}$, where $a_{0}$ is the Bohr radius for the ions. $\eta$ rewritten as $\eta=2 \pi \lambda_{d}^{2} / a^{2}$, is seen to be proportional to the squared ratio of the de Broglie thermal wavelength to the ion sphere radius and thus provides an appropriate gauge for quantum effects.

The many-body $g(r)$ is computed here by averaging over the density matrix $e^{-\beta H}$ using Path integral Monte Carlo based on the identity

$$
e^{-\beta H}=\left[e^{-\beta H / M}\right]^{M}
$$

where $M$ is an arbitrary integer. Insertion of complete sets of states between the $M$ factors on the right hand side of this equation leads to the usual path integral formulation of the density matrix, written here in real space,

$$
\left\langle\mathbf{R}\left|e^{-\beta H}\right| \mathbf{R}^{\prime}\right\rangle \equiv \rho\left(\mathbf{R}, \mathbf{R}^{\prime} ; \beta\right)=\int \ldots \int \rho\left(\mathbf{R}, \mathbf{R}_{1} ; \tau\right) \ldots \rho\left(\mathbf{R}_{M-1}, \mathbf{R}^{\prime} ; \tau\right) d \mathbf{R}_{1} \ldots d \mathbf{R}_{M-1}
$$



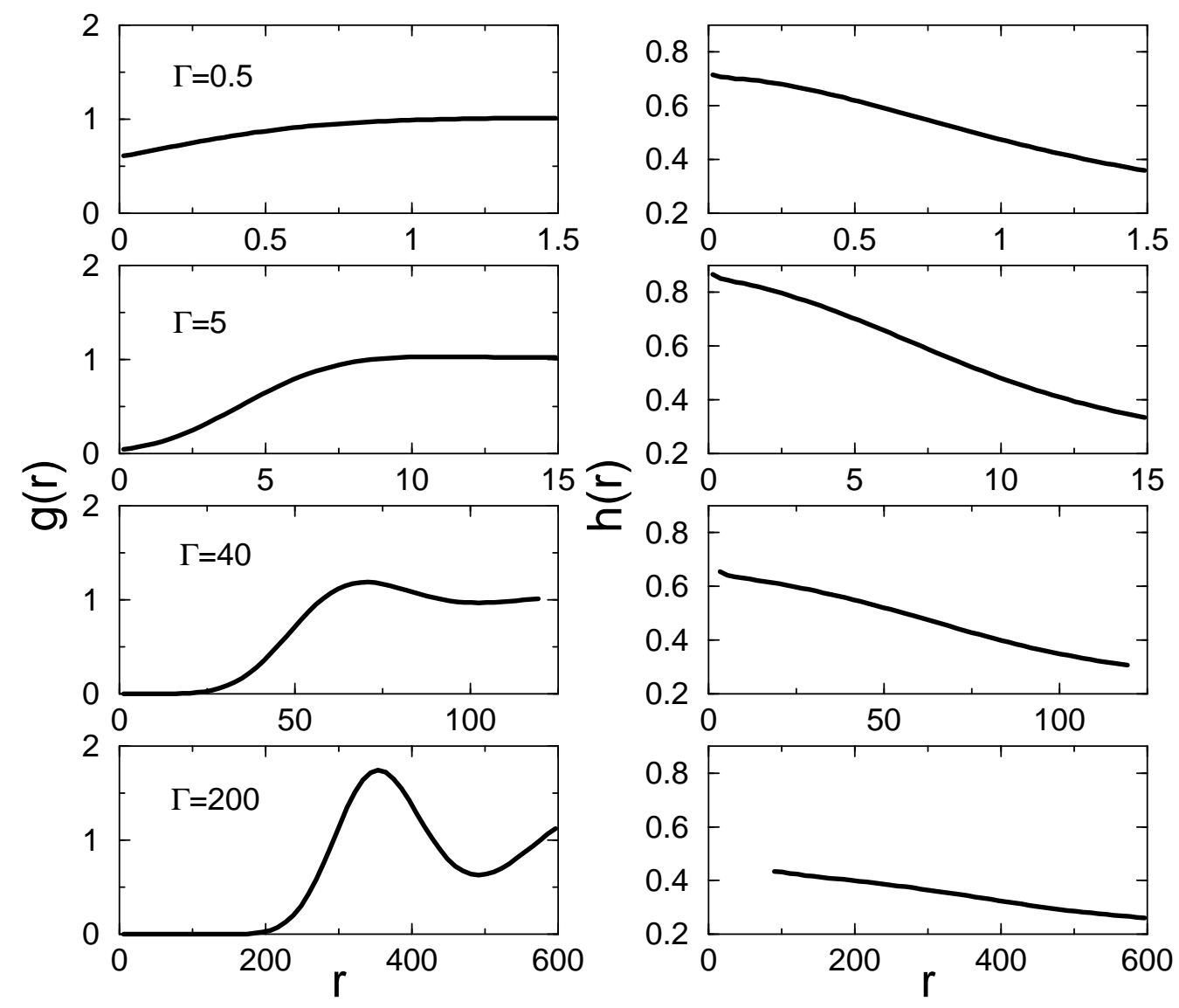

FIG. 2: Pair correlation functions $g(r)$ and the corresponding many-body enhancement $h(r)$ are shown as a function of the coupling parameter, $\Gamma$, for quantum parameter, $\eta=1 . r$ is in units of the nuclear Bohr radius.

with $\tau=\beta / M$. Each of the $M$ steps in the path now has a high temperature density matrix $\rho\left(\mathbf{R}_{k}, \mathbf{R}_{k+1} ; \tau\right)$ associated with it. The integrals are evaluated by Monte Carlo methods. First applied to realistic systems in reference 12 , the implementation details may be found in recent reviews 13 . In the results presented here the high temperature density matrix was taken as a product of exact pair density matrices. Typical $M$ values of 10 to 400, depending on $\beta$, gave a discretization error well below the Monte Carlo statistical uncertainties.

Typical examples of $g(r)$ and $h(r)=H(r) / \Gamma$ are shown in Fig. 2. Since $g(0)$ is now finite the $h(r)$ curves are considerably easier to extrapolate to the origin than for the classical OCP. A detailed discussion of the formal properties of $g(r)$ at small $r$ is given in section III and the appendix. $g(0), g_{b i n}(0)$ and $h(0)$ are given in table I for a range of $\Gamma$ and $\eta$ and are displayed in Fig. 3. 
TABLE I: Summary of OCP contact probabilities. The coupling parameter $\Gamma=\beta / r_{s}$ and quantum parameter $\eta=\Gamma / r_{s}$, or equivalently $\beta$ and $r_{s}$, are listed in columns one through four. The many-body enhancement factor, $h(0)$, is defined from the contact probability, $g(0)=g_{b i n}(0) e^{H(0)}=e^{-P(0)+\Gamma h(0)}$ (columns five through seven).

\begin{tabular}{|c|c|r|r|l|l|l|}
\hline$\eta$ & $\Gamma$ & $\beta$ & $r_{s}$ & $-\ln [g(0)]$ & $P(0)$ & $h(0)$ \\
\hline 0.1 & 0.5 & 2.5 & 5.0 & $2.205(18)$ & 2.638 & $0.87(4)$ \\
0.1 & 1.0 & 10 & 10 & $4.06(3)$ & 5.014 & $0.95(3)$ \\
0.1 & 2.0 & 40 & 20 & $7.22(6)$ & 9.243 & $1.01(3)$ \\
0.1 & 5.0 & 250 & 50 & $14.61(12)$ & 19.77 & $1.03(2)$ \\
0.1 & 10 & 1000 & 100 & $23.6(3)$ & 33.79 & $1.02(3)$ \\
0.1 & 40 & 16000 & 400 & $57.4(14)$ & 93.61 & $0.91(4)$ \\
\hline 0.25 & 0.5 & 1.0 & 2.0 & $1.292(12)$ & 1.707 & $0.82(2)$ \\
0.25 & 1.0 & 4.0 & 4.0 & $2.37(3)$ & 3.289 & $0.92(3)$ \\
0.25 & 2.0 & 16 & 8.0 & $4.25(5)$ & 6.192 & $0.97(3)$ \\
0.25 & 5.0 & 100 & 20 & $8.59(12)$ & 13.50 & $0.98(2)$ \\
0.25 & 10 & 400 & 40 & $14.33(3)$ & 23.90 & $0.957(3)$ \\
0.25 & 40 & 6400 & 160 & $34.3(16)$ & 67.32 & $0.83(4)$ \\
0.25 & 100 & 40000 & 400 & $59(4)$ & 129.5 & $0.71(4)$ \\
\hline 0.50 & 0.5 & 0.5 & 1.0 & $0.831(7)$ & 1.218 & $0.773(14)$ \\
0.50 & 1.0 & 2.0 & 2.0 & $1.504(19)$ & 2.373 & $0.869(19)$ \\
0.50 & 2.0 & 8.0 & 4.0 & $2.68(5)$ & 4.530 & $0.92(2)$ \\
0.50 & 5.0 & 50 & 10 & $5.53(12)$ & 10.17 & $0.93(2)$ \\
0.50 & 10 & 200 & 20 & $9.32(14)$ & 18.01 & $0.869(14)$ \\
0.50 & 40 & 3200 & 80 & $23.1(6)$ & 52.45 & $0.734(16)$ \\
0.50 & 100 & 20000 & 200 & $40(2)$ & 101.3 & $0.61(2)$ \\
\hline 1.0 & 0.5 & 0.25 & 0.5 & $0.514(4)$ & 0.8683 & $0.708(9)$ \\
1.0 & 1.0 & 1.0 & 1.0 & $0.917(11)$ & 1.704 & $0.787(11)$ \\
1.0 & 2.0 & 4.0 & 2.0 & $1.60(3)$ & 3.289 & $0.844(14)$ \\
1.0 & 5.0 & 25 & 5.0 & $3.29(11)$ & 7.540 & $0.85(2)$ \\
1.0 & 10 & 100 & 10 & $5.53(19)$ & 13.50 & $0.797(19)$ \\
1.0 & 40 & 1600 & 40 & $14.8(6)$ & 40.31 & $0.637(15)$ \\
1.0 & 200 & 40000 & 200 & $41(3)$ & 129.5 & $0.442(14)$ \\
1.0 & 400 & 160000 & 400 & $63(3)$ & 210.0 & $0.369(8)$ \\
1.0 & 600 & 360000 & 600 & $83(3)$ & 277.7 & $0.324(4)$ \\
\hline 2.0 & 0.5 & 0.125 & 0.25 & $0.3083(9)$ & 0.6175 & $0.6184(18)$ \\
2.0 & 1.0 & 0.5 & 0.5 & $0.536(5)$ & 1.218 & $0.682(5)$ \\
2.0 & 2.0 & 2.0 & 1.0 & $0.923(14)$ & 2.373 & $0.725(7)$ \\
2.0 & 5.0 & 12.5 & 2.5 & $1.86(6)$ & 5.545 & $0.737(13)$ \\
2.0 & 10 & 50 & 5.0 & $3.19(16)$ & 10.17 & $0.698(16)$ \\
2.0 & 40 & 800 & 20 & $9.1(5)$ & 30.92 & $0.546(12)$ \\
2.0 & 100 & 5000 & 50 & $17.0(8)$ & 61.34 & $0.443(8)$ \\
\hline
\end{tabular}

A clear reduction in $h(0)$ from the classical value (solid circles), which becomes more important as $\eta$ increases, is seen in Fig. 3. This yields reaction rates orders of magnitude smaller than would be predicted by the classical value for $h(0)$ at large $\Gamma$.

Fig. 10 1provides the basis for an intuitive understanding of this reduction due to quantum effects. $P(r)=-\ln g_{b i n}(r)$ can be roughly viewed as proportional to an "effective" quantum pair potential. Increasing $\eta$ means that the near neighbors of the reacting pair, located approximately one ion sphere radius away, are within a de Broglie thermal wavelength. Their effective quantum pair potential is then much less than the Coulomb potential as seen from the top panel of Fig. 1. This reduced repulsion lessens the many-body enhancement for the quantum system compared to that of a classical system at the same $\Gamma$. Reduction from the classical Coulomb value for $h(0)$ is also seen for classical screened Coulomb systems 14 and for quantum screened Coulomb systems (section IV). The physical explanation is again the reduced effective repulsion between the reacting pair and its surrounding neighbors due either to screening or quantum effects or both. This is a common pattern. A softer effective potential, either from quantum effects or screening enhances the two body contribution to $g(0)$ but reduces the many-body contribution.

Although $h(0)$ provides a compact way of presenting the data, the full $g(0)$, shown in Fig. U gives a more intuitive, physical picture. Fig. 4 shows the transition from the thermonuclear, temperature dependent regime, at low $\Gamma$ (high temperature) to the pycnonuclear, density dependent ground state regime at large $\Gamma$. The low temperature limit seems to be reached when the de Broglie thermal wavelength is of the order of $1 / 3$ the ion sphere radius. The principle feature of this graph is the smooth behavior of $g(0) . g(0)$ is seen to be a increasing function of the temperature 


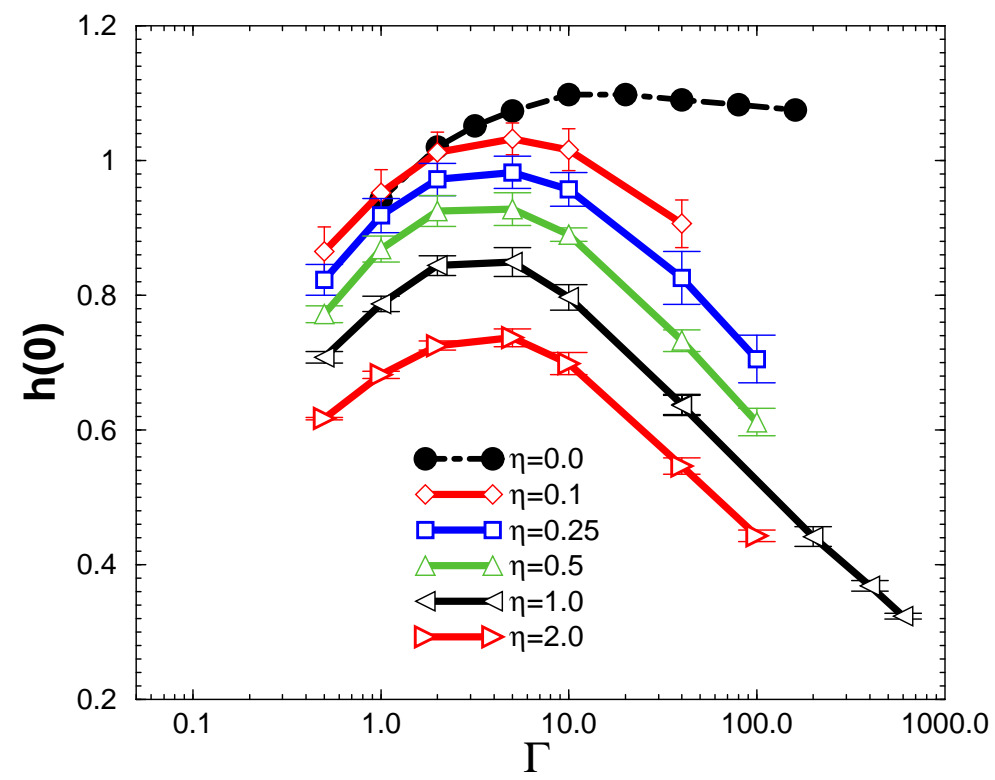

FIG. 3: The many-body enhancement factor $h(0)$ as a function of $\Gamma$ and quantum parameter $\eta$. Results for the classical OCP, $\eta=0$, are from reference ${ }^{3}$.

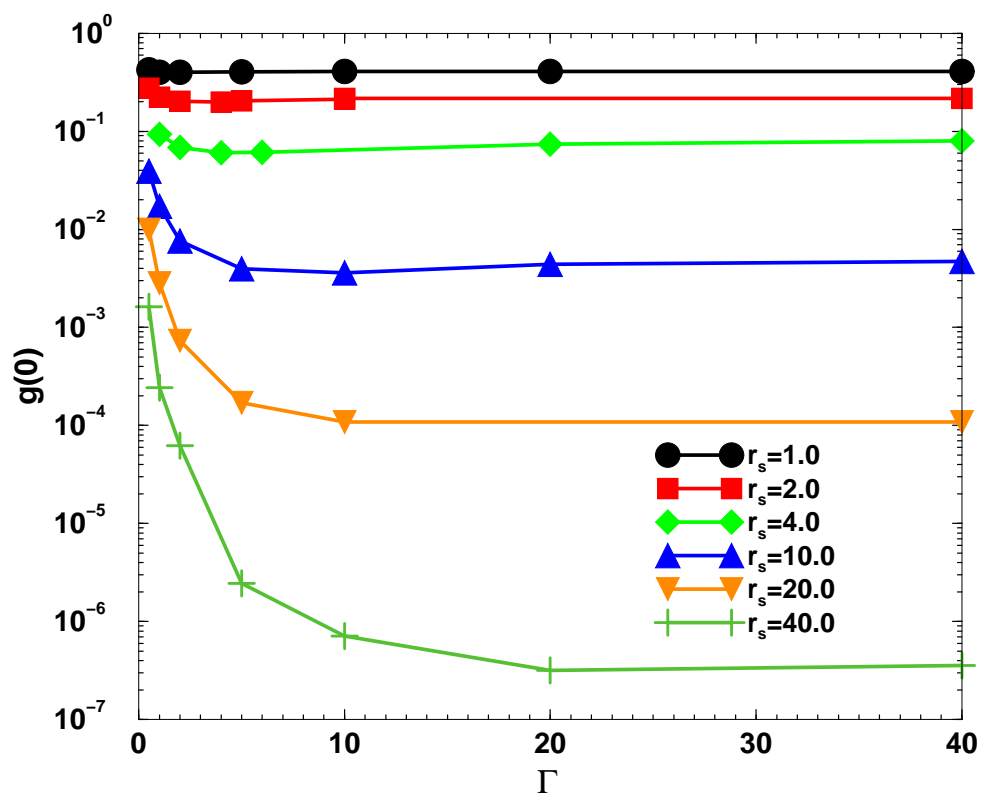

FIG. 4: Contact probabilities, $g(0)$, versus $\Gamma$ for indicated $r_{s}$ showing the transition from thermonuclear (strong temperature dependence, low $\Gamma$ ) to pycnonuclear (temperature independent) regimes.

and the density. For this model there are no peculiar combinations of density and temperature where the contact probability, and by implication the reaction rate, has an unexpected local maximum. 

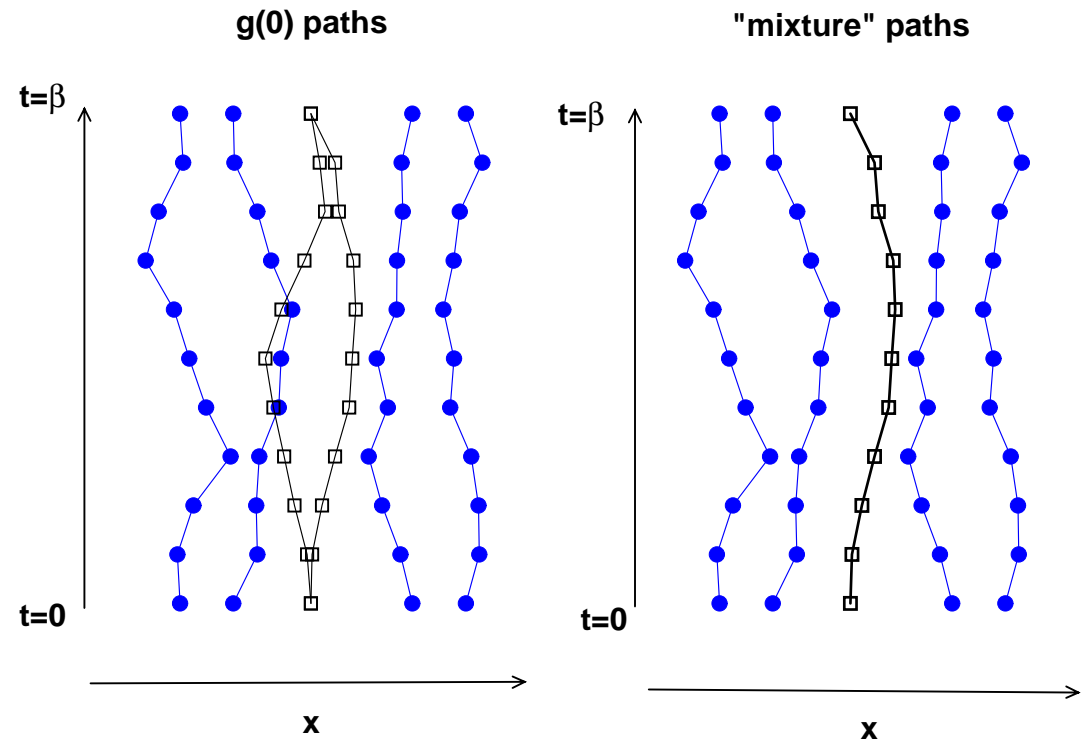

FIG. 5: Intuitive illustration of the relationship between $g(0)$ and the "mixture" free energy for a quantum system and its classical limit. The left panel shows a typical set of discretized (10 steps) paths which would enter the calculation of $g(0)$ for a six particle system. The nodes for the paths of the two "reacting" particles (in center) are shown as open squares. Nodes of the surrounding particle paths are shown as filled circles. Because of the delta function in the definition of $g(0)$ the two paths for the reacting pair overlap at imaginary times $t=0$ and $\beta$. The right panel shows typical paths for the density matrix, integrated to get the free energy, of a five particle mixture where the particle in center (nodes shown as open squares) has a mass and charge equal to the combined mass and charge of the reacting particles in the left panel. In the classical limit, when the de Broglie thermal wavelength goes to zero, the paths for the reacting pair (left panel) or mixture particle (right panel) become straight vertical lines. The potential energy at the reacting pair or mixture particle due to the four neighbors is then the same for both systems implying that $g(0)$ can be derived from the free energy difference.

\section{III. $g(r)$ AT SMALL $r$ AND RELATION TO FREE ENERGIES}

The radial distribution function may be expanded as

$$
g(r) \equiv \frac{\Omega}{N^{2}}\left\langle\sum_{i \neq j} \delta\left(\mathbf{r}-\mathbf{r}_{i j}\right)\right\rangle=g(0)+C r^{2}+O\left(r^{4}\right) .
$$

In a classical system the coefficient of the $r^{2}$ term is proportional to mean squared force on the two fused particles. $g(0)$ is related to the difference between the free energy of a mixture consisting of the fused pair and the N-2 other particles and the free energy of the original system ${ }^{15}$. For a quantum system the expansion coefficients are more complicated.

Since the delta function in the definition of $g(0)$ causes the two "reacting" particles to overlap it might be thought that the relation to the hypothetical mixture free energy which holds in the classical case would be sufficient in general. For those comfortable with the path integral ideas used in this paper the cartoon in Fig. 5 may give an intuitive understanding of why this is not true. Others are relegated to the detailed derivation in the appendix.

The two panels of this cartoon show the paths (discretized here into 10 segments) that contribute to $g(0)$ for a six particle system (left panel) and the paths contributing to the density matrix of a mixture of five particles (right panel) where the two "reacting" particles have been fused into one particle with the combined charge and mass of the reacting particles. In the figure the nodes in the discretized paths of the reacting (or fused) pair are shown as open squares. The obvious difference in the paths is that in the $g(0)$ case the delta function in the definition causes the paths of the reacting particles to overlap at imaginary times $t=0$ and $t=\beta$ but not at other times. In the mixture, by contrast, there is only one path for the fused particle. Since this fused particle has the combined mass of the reacting pair it would be a "more classical" particle and typically have a less fluctuating path.

The scale for the deviation of these paths from straight vertical lines is set by the de Broglie thermal wavelength. The different contribution from the two cases (left and right panel) comes from the potential energy around the paths of the reacting (or fused) pair due its neighbors. In the classical limit the de Broglie thermal wavelength goes to zero and the paths reduce to lines. The potential energy of the other particles at the reacting pair (left panel) is then the 


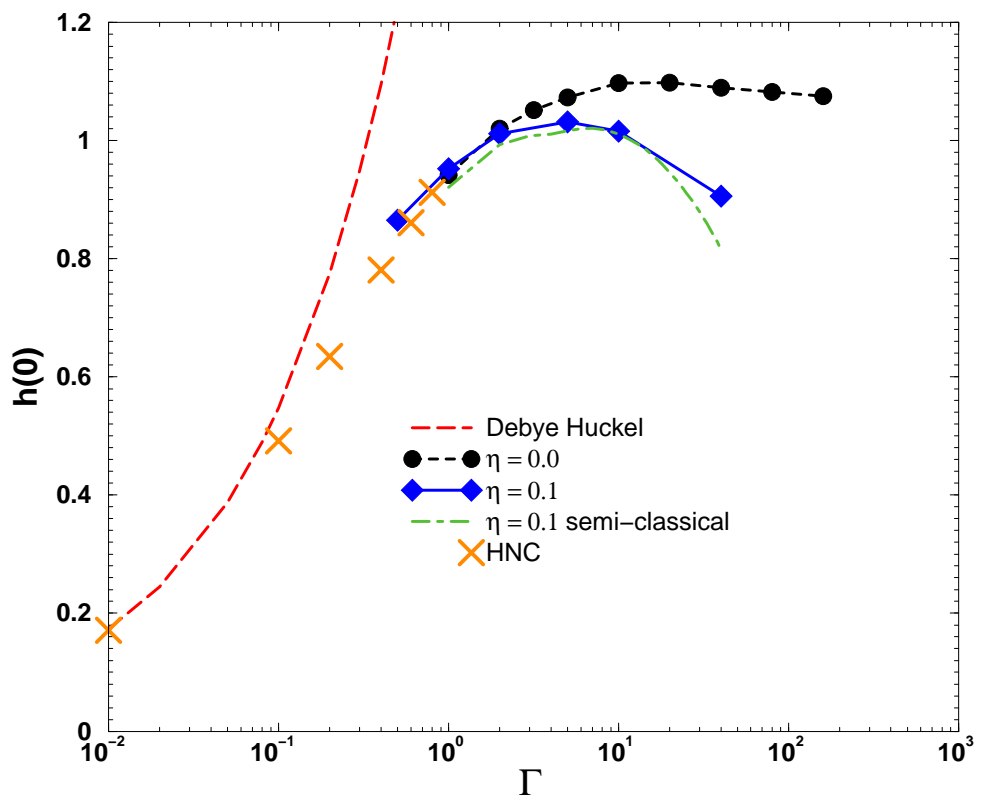

FIG. 6: Debye-Hückel approximation for $h(0)$ (dashed line) as low $\Gamma$ limit for the classical, $\eta=0$, system. PIMC (solid diamonds) and semiclassical values (dot-dashed line), Eq. 10, for $h(0)$ at $\eta=0.1$ are also shown. Hypernetted chain integral equation results (crosses) are from Ref. 18

same as for the fused particle in the mixture case (right panel). Hence the correspondence in the classical limit. This also indicates that the classical relation between $g(0)$ and the free energy difference holds when the reacting particles, but not necessarily the other neighbors, are treated classically.

Details for the $g(r)$ expansion are given in the appendix. It follows the original work of Jancovici 16 and Alastuey and Jancovici17. We differ in explicitly separating the $g_{\text {bin }}(0)$ term, which is given numerically by Eq. 3 , and also the free energy difference, approximations for which may be available from other theories. This involves a slight rearrangement of terms.

To lowest order in the quantum parameter $\eta$ and $r$,

$$
H(r)=-\beta[F(1, N-2)-F(0, N)]-\frac{\Gamma}{4 a^{2}}\left\langle r^{2}\right\rangle
$$

where the first argument in the interaction free energies, $F$, denotes the number of combined mass, combined charge ions. $\left\langle r^{2}\right\rangle$ is calculated from the relative pair density matrix

$$
\left\langle r^{2}\right\rangle(r, \beta) \equiv \frac{1}{\beta} \int_{0}^{\beta} d s \int d \mathbf{r}^{\prime} \frac{\rho\left(\mathbf{r}, \mathbf{r}^{\prime} ; \beta-s\right) r^{\prime 2} \rho\left(\mathbf{r}^{\prime}, \mathbf{r} ; s\right)}{\rho(\mathbf{r}, \mathbf{r} ; \beta)}
$$

In the classical limit $\left\langle r^{2}\right\rangle=r^{2}$ and the usual result

$$
H(r)=-\beta[F(1, N-2)-F(0, N)]-\frac{\Gamma}{4 a^{2}} r^{2}
$$

is regained. Using the Debye-Hückel free energies 18 in Eq. 9 gives the classical weak coupling limit, $h(0)=\sqrt{3 \Gamma}$. As suggested in Fig. 6 the classical values will approach this for $\Gamma \leq 0.1$. Although simulation results for the classical $h(0)$ at $\Gamma<1$ have not been published the approach to the Debye-Hückel limit is clearly seen in calculations based on the $\mathrm{HNC}$ approximation ${ }^{19}$.

More generally Eq. 8 may be written as

$$
\left\langle r^{2}\right\rangle(r, \beta) \equiv r^{2}+\alpha(r, \beta) \frac{\hbar^{2} \beta}{m}
$$

where the defined function $\alpha(r, \beta)$, multiplying the free particle result, tends to 1 as $\beta \rightarrow 0 . \alpha(0, \beta)$ is tabulated in Table III of the appendix. Using Eq. [10] together with the first order Wigner-Kirkwood quantum correction for the 


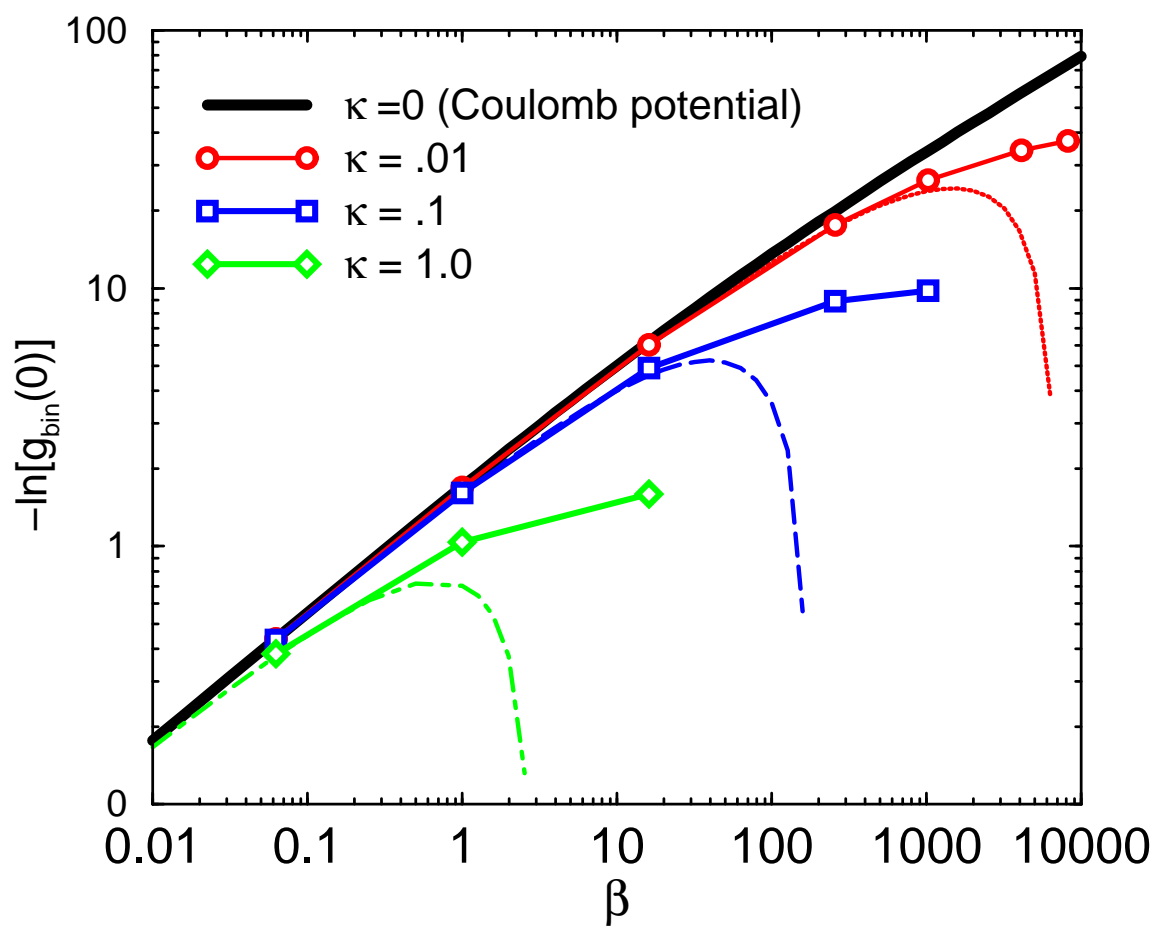

FIG. 7: $g_{b i n}(0)$ for the screened Coulomb potential at $\kappa=100$ (open circles), 10 (open squares), and 1 (open diamonds) compared to pure Coulomb result (thick solid line) and "energy shift" approximation $g_{\text {bin }}^{\kappa}(0)=e^{\beta \kappa} g_{b i n}^{\kappa=0}(0)$.

free energy ${ }^{20}$, Eq. 7 gives the lowest order quantum correction to the enhancement factor

$$
h(0)[\Gamma, \eta]-h(0)[\Gamma, 0] \approx-\frac{\eta}{4}\left[\alpha\left(0, \Gamma^{2} / \eta\right)-1 / 2\right] .
$$

The result is shown for $\eta=0.1$ in Fig. 6 and qualitatively reproduces the PIMC results showing significant reduction from the classical $h(0)$.

\section{ELECTRON SCREENING EFFECTS}

The OCP model discussed above is justified when the electrons are sufficiently degenerate to not respond to the ionic potential. Small deviations from this may be treated by linear response leading to an effective ion-ion potential. The most common such potential is the screened Coulomb potential

$$
V(r)=\frac{e^{-\kappa r}}{r}
$$

with screening length $1 / \kappa$, which we consider in this section.

From the Feynman-Kac formula applied to the pair density matrix for $r=0$,

$$
\frac{\rho_{b i n}(0,0 ; \beta)}{\rho_{\text {bin }}^{\text {free }}(0,0 ; \beta)}=\left\langle e^{-\int_{0}^{\beta} V[r(s)] d s}\right\rangle_{B M P}
$$

where the angular brackets denote an average over all Brownian motion paths beginning and ending, after a time $\beta$, at the origin. If the screened Coulomb potential is approximated as

$$
\frac{e^{-\kappa r}}{r} \approx \frac{1}{r}-\kappa
$$

for $r \leq 1 / \kappa$ then Eq. 13 becomes

$$
\frac{\rho_{b i n}(0,0 ; \beta)}{\rho_{b i n}^{f r e e}(0,0 ; \beta)}=e^{\beta \kappa}\left\langle e^{-\int_{0}^{\beta} \frac{1}{r(s)} d s}\right\rangle_{B M P}
$$




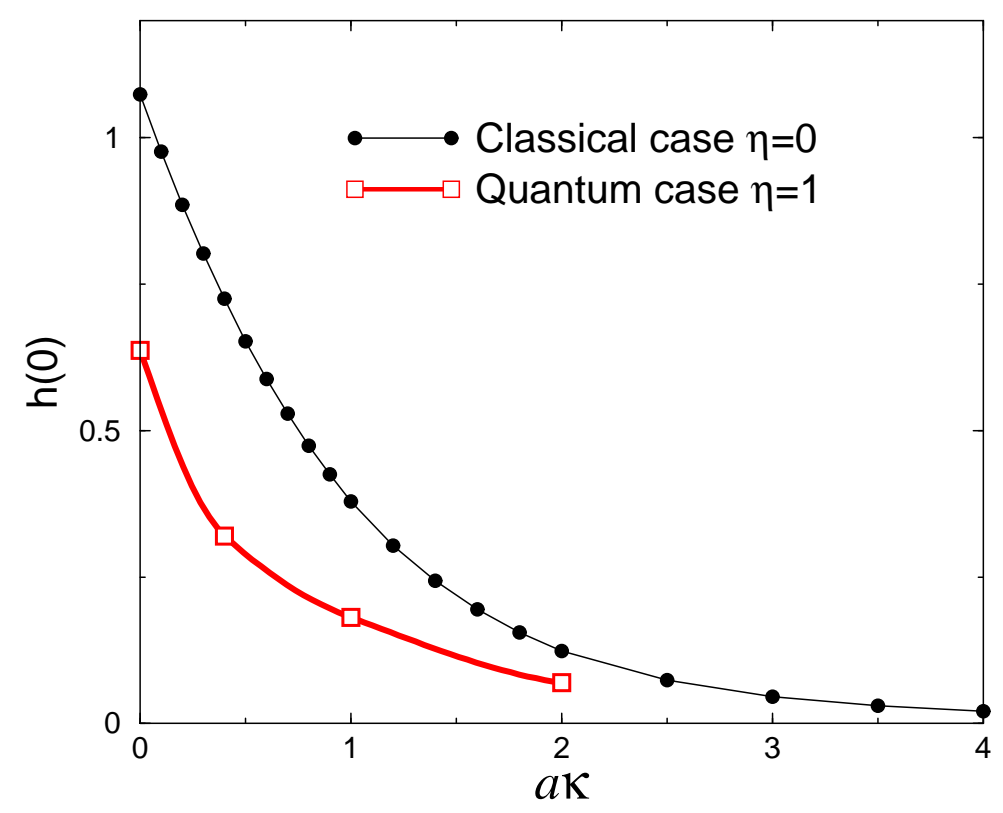

FIG. 8: (color online) $h(0)$ for the classical ${ }^{14}, \eta=0$, and quantum, $\eta=1$, Yukawa system at $\Gamma=40$ as a function of the inverse screening length $\kappa$ times ion sphere radius $a . \kappa=0$ corresponds to the unscreened Coulomb potential results in Fig. 3.

or $g_{b i n}^{\kappa}(0)=e^{\beta \kappa} g_{\text {bin }}^{\text {Coulomb }}(0)$. The first factor may be interpreted as an energy shift $\Delta E=-\kappa$. This "constant energy shift" approximation fails when the de Broglie thermal wavelength, which gives a scale for the extent of the Brownian motion paths, is larger than the screening length, $1 / \kappa$, so the paths sample regions where Eq. 14 does not apply.

Fig. 7 shows $g_{b i n}(0)$ for several $\kappa$ values. These were computed here by rewriting the Feynman-Kac formula as

$$
\frac{\rho_{b i n}(0,0 ; \beta)}{\rho_{b i n}^{\kappa=0}(0,0 ; \beta)}=\left\langle e^{-\int_{0}^{\beta}(V[r(s)]-1 / r(s)) d s}\right\rangle_{C M P}
$$

where now the difference between the screened and unscreened Coulomb potential is integrated over paths distributed according to the unscreened Coulomb potential density matrix (CMP).

The dashed lines show the corresponding constant energy shift approximation. This constant energy shift approximation is valid in most astrophysical applications ${ }^{8}$ but, as seen in Fig. 7 , it can dramatically overestimate $g_{b i n}(0)$ at low temperatures giving very misleading, excessive reaction rates 21 .

Turning now to many-body effects, screening has been shown to reduce $h(0)$ in the classical OCP14. Its effect in the quantum OCP, shown in Fig. 8, is similar. The reduced repulsion from surrounding ions due to screening again reduces the enhancement effect.

\section{QUANTUM OCP THERMODYNAMIC PROPERTIES}

The PIMC calculations of the density matrix used to obtain $g(r)$ also yield the kinetic and potential energies. Results for the computations used in this paper are given in Table II.

The PIMC and harmonic approximation values for the kinetic energy

$$
K=\frac{1}{2} \sum_{k, j} \hbar \omega_{j}(\mathbf{k})\left[\frac{1}{e^{\beta \hbar \omega_{j}(\mathbf{k})}-1}+\frac{1}{2}\right]
$$

where $\omega_{j}(\mathbf{k})$ are the vibrational frequencies of the BCC Wigner lattice 24 are compared in the table. Even though all simulations tabulated were in the fluid phase the agreement at higher $\Gamma$ is quite good. This is not entirely surprising as similar agreement with other properties has been often noted for the purely classical system due to the long range nature of the interaction. The harmonic approximation also gives the correct lowest order quantum correction,

$$
K=\frac{3}{2} k T+\frac{1}{8} \eta^{2} / \Gamma
$$




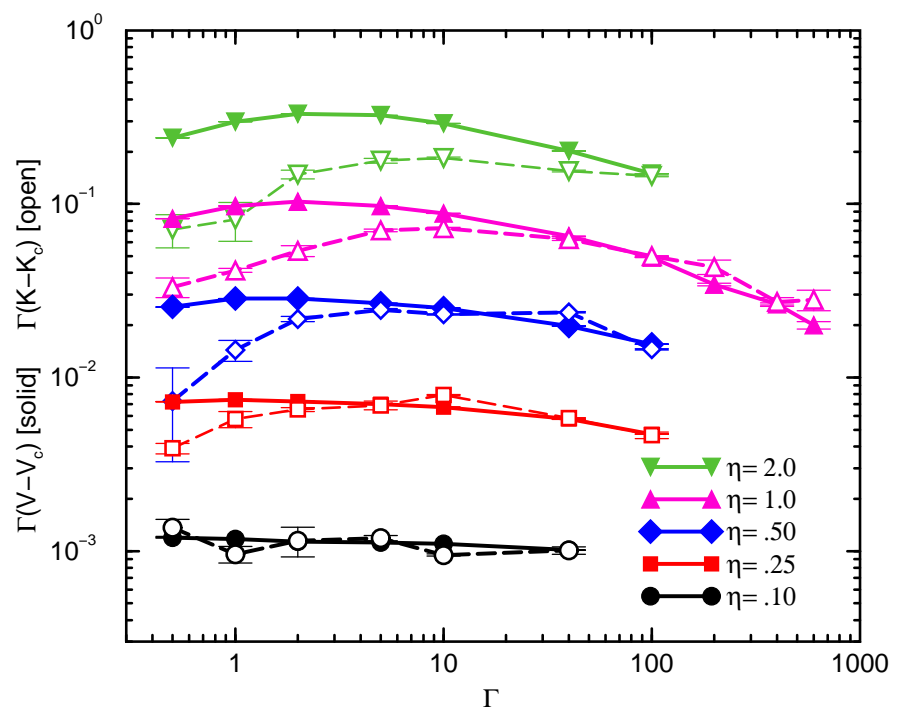

FIG. 9: (color online) Comparison of the quantum kinetic, $K-K_{c}$ (open symbols and dashed lines), and potential energy, $V-V_{c}$ (solid symbols and lines) per particle (data from Table II).

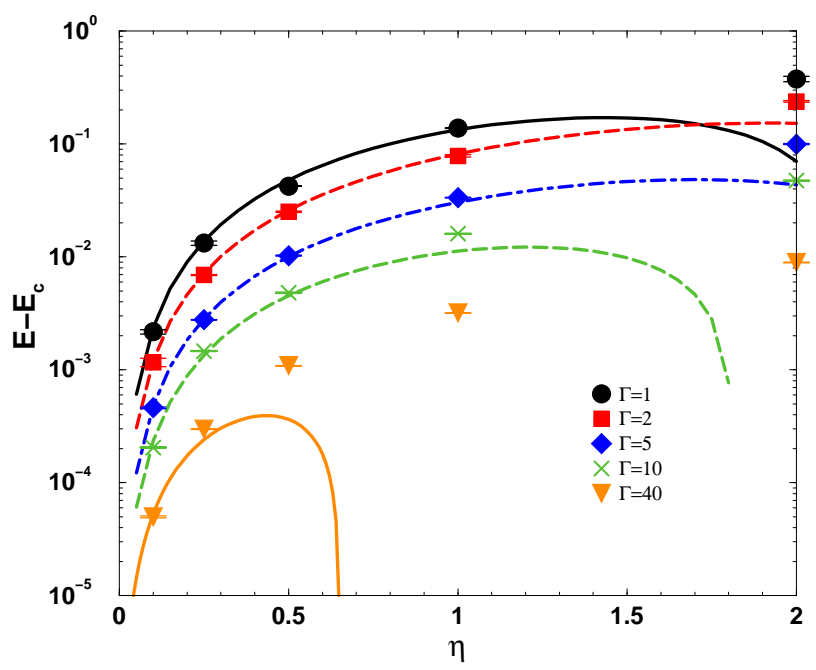

FIG. 10: (color online) Comparison of the quantum energy per particle, $E-E_{c}$ for $\Gamma=1$ (solid circles), $\Gamma=2$ (solid squares) $\Gamma=5$, (solid diamonds), $\Gamma=10$ (crosses) and $\Gamma=40$ (solid triangles) with the second order Wigner-Kirkwood ( $\hbar$ expansion) correction (corresponding solid or dashed lines).

using the Kohn sum rule. As expected for fixed $\Gamma$ this agreement worsens as $\eta$ increases.

The quantum corrections to the kinetic and potential energy as functions of $\Gamma$ and $\eta$ are plotted in figure 9 . As expected the magnitude of the quantum corrections for both quantities increases with $\eta$. At high $\Gamma$ the quantum kinetic and potential energies are seen to converge. Again, if the harmonic approximation where theses quantum corrections are equal, gives a good description of the thermodynamics even in the liquid state at high $\Gamma$, then this convergence is understandable. The slower convergence as $\eta$ increases for a fixed $\Gamma$ is also understandable since anharmonic corrections would be larger here.

The quantum contributions to the total energy are compared to the expression for the second order Wigner Kirkwood correction as given by Hansen and Viellefosse $e^{25}$ in Table II and in Fig. 10. Similar limits on the convergence of this second order approximation were previously seen by Jones and Ceperley ${ }^{23}$. (See their figure 3.) 
TABLE II: Excess quantum kinetic $\left(K-K_{c}\right)$, "excess" potential $\left(V-V_{c}\right)$, and internal $(E)$ energy per particle from PIMC with 54 distinguishable particles and $M$ time slices. $K_{c}=3 / 2 \beta$ and $V_{c}$ is the potential energy for the classical OCP with 54 particles in the periodic cell, taken as $\beta V_{c}=\{-0.24497,-0.57994,-1.32795,-3.7621,-7.9997,-34.2373\}$ for $\Gamma=\{0.5,1,2,5,10,40\}$ and as given by the $\mathrm{fit}^{25} \beta V_{c}=-0.899375 \Gamma+0.569333 \Gamma^{1 / 3}-0.224470-0.017875 / \Gamma^{1 / 3}$ for larger $\Gamma$ values. The subscript $h$ denotes values from the harmonic approximation for the BCC Wigner lattice. The first and second order Wigner Kirkwood corrections to the energy from reference 25 are given in the last column

\begin{tabular}{|c|c|c|c|c|c|c|c|c|c|c|c|}
\hline$\eta$ & $\bar{\Gamma}$ & $\beta$ & $r_{s}$ & $M$ & $K-K_{c}$ & $K_{h}-K_{c}$ & $\bar{V}$ & $V-V_{c}$ & $E$ & $E-E_{c}$ & $\overline{\mathrm{WK}}$ \\
\hline 0.1 & 0.5 & 2.5 & 5 & 25 & $0.0019(8)$ & 0.0023 & $-0.09557(3)$ & $.00239(3)$ & $0.5063(8)$ & $0.0043(8)$ & 0.00454 \\
\hline 0.1 & 1 & 10 & 10 & 10 & $0.0010(1)$ & 012 & $.056824(5)$ & $01170(5)$ & $0.0941(1)$ & $0022(1)$ & .00238 \\
\hline 0.1 & 2 & 40 & 20 & 40 & (1) & 61 & 4) & 1) & l) & $2(1)$ & 00121 \\
\hline 0.1 & 5 & 250 & 50 & 25 & $0.000238(8)$ & 000244 & $-0.0148247(7)$ & $0002235(7)$ & $-0.008587(8)$ & $000462(8)$ & .000480 \\
\hline 0.1 & 10 & 1000 & 100 & 10 & $0.000095(1)$ & 00012 & $-0.0078895(1)$ & $.0001102(1)$ & $-0.006295(1)$ & $0.000205(1)$ & .000236 \\
\hline 0.1 & 40 & 16000 & 400 & 80 & $0.000025(1)$ & 0.000028 & $-0.00211452(9$ & $.0000253(1)$ & $-0.001996(1)$ & $0.000050(1)$ & 0.000053 \\
\hline 0.25 & 0.5 & .0 & 2 & 10 & $(5)$ & 15 & (3) & $493(3)$ & $1.2773(5)$ & $0.0223(5)$ & 412 \\
\hline 0.25 & 1 & 4.0 & 4 & 40 & $0.0058(6)$ & 077 & $13753(3)$ & $00745(3)$ & $0.2432(6)$ & $0.0133(6)$ & 1381 \\
\hline 0.25 & 2 & 16 & 8 & 16 & $0.00327(9)$ & 0382 & $.079355(4)$ & $03642(4)$ & $0.01766(8)$ & $0.00691(8)$ & .00713 \\
\hline 25 & 5 & 100 & 10 & 80 & (8) & 015 & $20(4)$ & $01(4)$ & -0.0 & & .002819 \\
\hline 25 & 10 & 400 & 40 & 40 & ( $(7)$ & 727 & & & & $65(7)$ & 348 \\
\hline 0.25 & 40 & 6400 & 160 & 80 & $6(2)$ & 000156 & $2058(1)$ & $1438(1)$ & $-0.004826(2)$ & $290(2)$ & 00241 \\
\hline 0.25 & 100 & 40000 & 400 & 200 & $0.000047(2)$ & .000051 & $-0.00213982(9)$ & $.0000472(1)$ & $-0.002056(2)$ & $0.000094(2)$ & 0.000022 \\
\hline 50 & 0.5 & 5 & 1 & 50 & & 0 & $89(1)$ & $099(1)$ & $6(8)$ & 0.0 & 798 \\
\hline 0 & 1 & 2.0 & 2 & 50 & & & & & & (2) & 797 \\
\hline 0.50 & 2 & 8.0 & 4 & 40 & $9(4)$ & & (1) & & $0.0466(4)$ & 1(4) & 2582 \\
\hline 50 & 5 & 50 & 10 & 50 & $0.00491(9)$ & 82 & $-0.069885(4)$ & $356(4)$ & $-0.03497(9)$ & 1027(9) & 01005 \\
\hline 50 & 10 & 200 & 20 & 50 & & & & & & & 53 \\
\hline 0.50 & 40 & 00 & 80 & 80 & 5) & 541 & 6) & & -0 & $088(5)$ & 00364 \\
\hline 0.50 & 100 & 20000 & 200 & 100 & $0.000145(2)$ & 0.000166 & $42185(2)$ & $1555(2)$ & $-0.003998(2)$ & $0.000301(2)$ & -0.000452 \\
\hline 1.0 & 0.5 & 0.25 & 0.5 & 25 & ) & 5 & ) & & (9) & $\overline{(9)}$ & .04381 \\
\hline 1.0 & 1 & & 1 & 10 & & & & $2(6)$ & & & 338 \\
\hline 1.0 & 2 & .0 & 2 & 40 & 2) & & 5) & (5) & & (2) & 316 \\
\hline 1.0 & 5 & 2 & 5 & 25 & $0.0141(2)$ & & & $17(2)$ & $-0.0569(2)$ & & 044 \\
\hline 1.0 & 10 & & 10 & 80 & $0.00723(9)$ & & & $8827(4$ & $-0.04894(9)$ & $0.01606(9)$ & 112 \\
\hline 1.0 & 40 & 0 & 40 & 12 & & & & 7 & -0. & & 334 \\
\hline 1.0 & 100 & 00 & 100 & 100 & (2) & 52 & (2) & $905(7)$ & $-0.007606(2)$ & $0.000986(3)$ & 0611 \\
\hline 1.0 & 200 & 40000 & 200 & 400 & $0.00021(2)$ & 0.000198 & $-0.004248(3)$ & $00172(3)$ & $-0.00399(2)$ & $0.00038(2)$ & 0697 \\
\hline 1.0 & 400 & 0000 & 400 & 400 & $68(4)$ & 074 & $5(9)$ & $662(9)$ & $-0.002080(4)$ & $134(4)$ & -0.00735 \\
\hline 1.0 & 600 & 30000 & 600 & 450 & $0.000047(6)$ & 0.000041 & $-0.001453(2)$ & $0.0000334(2)$ & $-0.001402(7)$ & $0.000080(7)$ & -0.00746 \\
\hline 2.0 & 0.5 & 0.125 & 0.25 & 50 & & & & & $10.66(3)$ & & -1.6495 \\
\hline 2.0 & 1 & 0.5 & 0.5 & 50 & & & $5(3)$ & $74(3)$ & $2.22(2)$ & $(2)$ & 7017 \\
\hline 2.0 & 2 & & 1 & 5 & & & & & $25(4)$ & & 26 \\
\hline 2.0 & 5 & 12.5 & 2.5 & 50 & & & $522(5)$ & & & & 0.04349 \\
\hline 2.0 & 10 & 50 & 5 & 50 & $0.0184(3)$ & & $97(2)$ & $902(2)$ & $826(3)$ & $174(3)$ & -0.01006 \\
\hline 2.0 & 40 & 800 & 20 & 80 & $0.00387(5)$ & 0.00565 & $748(4)$ & $0.005049(4)$ & $-0.03201(5)$ & $0.00892(5)$ & -0.0517 \\
\hline 2.0 & 100 & 5000 & 50 & 100 & $0.00145(2)$ & 0.00158 & $-0.016003(2)$ & $0.001493(2)$ & $-0.01425(1)$ & $0.00294(1)$ & -0.0589 \\
\hline
\end{tabular}

\section{CONCLUSIONS}

In conclusion, quantum effects have been shown to significantly reduce the many-body enhancement factor which influences nuclear reaction rates in dense plasmas. Electron screening effects produce a further reduction. The contact probability and reaction rates based on it increase monotonically with temperature and density. The relation between the contact probability and free energy differences is derived and intuitively illustrated.

\section{APPENDIX A: SMALL $r$ EXPANSION OF $h(r)$ IN THE SEMICLASSICAL LIMIT}

In a classical plasma $g(0)$ can be related to a free energy difference ${ }^{2}$ and a simple, explicit value given for the coefficient of the $r^{2}$ expansion term ${ }^{16}$. For the quantum plasma no similar, exact relation has been found however an expansion in the ratio of the de Broglie thermal wavelength to the ion sphere radius for $g(r)$ at small $r$ can be made ${ }^{16}$. This expansion, which reduces to the classical result, is reviewed here. The principal results are given below 
in Eqs. A12 A17 A18 and in Table III for $\alpha(0 ; \beta)$.

Starting from the definition of the radial distribution function for a one component system

$$
g(\mathbf{r})=\Omega\left\langle\delta\left(\mathbf{r}-\mathbf{r}_{\mathbf{1 2}}\right)\right\rangle \equiv \Omega \frac{\operatorname{Tr}\left[e^{-\beta H} \delta\left(\mathbf{r}-\mathbf{r}_{\mathbf{1 2}}\right)\right]}{\operatorname{Tr}\left[e^{-\beta H}\right]}=\Omega \frac{\operatorname{Tr}\left[e^{-\beta H} \delta\left(\mathbf{r}-\mathbf{r}_{\mathbf{1 2}}\right)\right]}{\mathcal{Z}}
$$

where particles one and two have been singled out, the Hamiltonian

$$
H=\sum_{j=1}^{N} K_{j}+\sum_{k} \sum_{j<k} v_{j k}
$$

with $K$ the kinetic energy operator is rewritten in terms of center of mass and relative coordinates for the "reacting" particles, one and two, $\mathbf{R}=\left(\mathbf{r}_{1}+\mathbf{r}_{2}\right) / 2, \mathbf{r}_{\mathbf{1 2}}=\mathbf{r}_{1}-\mathbf{r}_{\mathbf{2}}$. Doing this, separating out the terms involving only particles one and two, and adding and subtracting a term representing the interaction of both particles one and two at the center of mass position with the "spectator" particles $3 \ldots N$ the Hamiltonian can be rewritten as,

$$
H=H_{r e l}(\mathbf{r})+H_{m i x}\left(\mathbf{R}, \mathbf{r}_{3} \ldots \mathbf{r}_{N}\right)+\Delta V\left(\mathbf{R}, \mathbf{r}, \mathbf{r}_{3} \ldots \mathbf{r}_{N}\right)
$$

The "relative" Hamiltonian

$$
H_{r e l}(\mathbf{r})=K_{r}+v(\mathbf{r})
$$

commutes with the "mixture" Hamiltonian

$$
H_{m i x}\left(\mathbf{R}, \mathbf{r}_{3} \ldots \mathbf{r}_{N}\right)=K_{R}+\sum_{j=3}^{N} K_{j}+\sum_{j=3}^{N} \sum_{k>j} v_{j k}+2 \sum_{j=3}^{N} v\left(\mathbf{R}-\mathbf{r}_{\mathbf{j}}\right)
$$

which corresponds to a particle of double the mass and charge at the center of mass position, $\mathbf{R}$, and the $N-2$ spectator particles. The coupling term

$$
\begin{aligned}
\Delta V\left(\mathbf{R}, \mathbf{r}, \mathbf{r}_{3} \ldots \mathbf{r}_{N}\right) & =\sum_{j=3}^{N}\left[v\left(\mathbf{r}_{1}-\mathbf{r}_{\mathbf{j}}\right)+v\left(\mathbf{r}_{\mathbf{2}}-\mathbf{r}_{\mathbf{j}}\right)-2 v\left(\mathbf{R}-\mathbf{r}_{\mathbf{j}}\right)\right] \\
& =\sum_{j=3}^{N}\left[v\left(\mathbf{R}+\frac{\mathbf{r}}{\mathbf{2}}-\mathbf{r}_{\mathbf{j}}\right)+v\left(\mathbf{R}-\frac{\mathbf{r}}{\mathbf{2}}-\mathbf{r}_{\mathbf{j}}\right)-2 v\left(\mathbf{R}-\mathbf{r}_{\mathbf{j}}\right)\right] \\
& =\sum_{j=3}^{N} \nabla \nabla v\left(\mathbf{R}-\mathbf{r}_{\mathbf{j}}\right): \mathbf{r r} / 4+O\left(\mathbf{r}^{4}\right)
\end{aligned}
$$

is the difference between the interactions of all other particles in the system with particles 1 and 2 at their actual positions minus these interactions when particles 1 and 2 are fused at their center of mass position.

Using Eq. A3 in the expression for $g(r)$ and taking the trace in real space

$$
g(r)=\frac{\Omega}{\mathcal{Z}} \int\left\langle\mathbf{R}, \mathbf{r}, \mathbf{r}_{\mathbf{3}}, \ldots \mathbf{r}_{\mathbf{N}}\left|e^{-\beta\left(H_{r e l}+H_{m i x}+\Delta V\right)}\right| \mathbf{R}, \mathbf{r}, \mathbf{r}_{\mathbf{3}}, \ldots \mathbf{r}_{\mathbf{N}}\right\rangle d \mathbf{R} d \mathbf{r}_{\mathbf{3}} \ldots d \mathbf{r}_{\mathbf{N}}
$$

When averaged over an isotropic system

$$
\nabla \nabla v\left(\mathbf{R}-\mathbf{r}_{\mathbf{j}}\right)=\nabla^{2} v\left(\mathbf{R}-\mathbf{r}_{\mathbf{j}}\right) \stackrel{\leftrightarrow}{\mathbf{I}}
$$

The Laplacian is easily evaluated for the Coulomb system considered here where the interaction, accounting for periodic boundary conditions and charge neutrality, is the Ewald potential, $\Psi_{\text {Ewald }}(r)$. Using $\nabla^{2} \Psi_{E w a l d}(r)=-4 \pi \delta(r)+4 \pi / \Omega$, where, physically, the constant comes from the neutralizing background,

$$
\Delta V\left(\mathbf{R}, \mathbf{r}, \mathbf{r}_{3} \ldots \mathbf{r}_{N}\right)=\frac{4 \pi Z^{2}(N-2)}{\Omega} \frac{r^{2}}{12}+O\left(r^{4}\right)=\frac{Z^{2} r^{2}}{4 a^{3}}+O\left(r^{4}\right) .
$$

The simplification that the coefficient of the lowest order term in $r$ is constant is unique to Coulomb systems.

With this expansion and using the fact that $H_{m i x}$ commutes with $H_{r e l}$ and the above lowest order term for $\Delta V$

$$
\begin{aligned}
g(r) & =\frac{\Omega}{\mathcal{Z}}\left\langle\mathbf{r}\left|e^{-\beta\left(H_{r e l}+C r^{2}\right)}\right| \mathbf{r}\right\rangle \int\left\langle\mathbf{R}, \mathbf{r}_{\mathbf{3}}, \ldots \mathbf{r}_{\mathbf{N}}\left|e^{-\beta H_{m i x}}\right| \mathbf{R}, \mathbf{r}_{\mathbf{3}}, \ldots \mathbf{r}_{\mathbf{N}}\right\rangle d \mathbf{R} d \mathbf{r}_{\mathbf{3}} \ldots d \mathbf{r}_{\mathbf{N}}+O\left(<r^{4}>\right) \\
& =\Omega e^{-\beta\left(F_{m i x}-F_{\text {pure }}\right)}\left\langle\mathbf{r}\left|e^{-\beta\left(H_{r e l}+C r^{2}\right)}\right| \mathbf{r}\right\rangle+O\left(<r^{4}>\right)
\end{aligned}
$$


where $C=Z^{2} / 4 a^{3}$. The free energies $F_{\text {pure }}, \mathcal{Z}=\exp \left(-\beta F_{\text {pure }}\right)$, and $F_{\text {mix }}$,

$$
e^{-\beta F_{m i x}}=\int\left\langle\mathbf{R}, \mathbf{r}_{\mathbf{3}}, \ldots \mathbf{r}_{\mathbf{N}}\left|e^{-\beta H_{m i x}}\right| \mathbf{R}, \mathbf{r}_{\mathbf{3}}, \ldots \mathbf{r}_{\mathbf{N}}\right\rangle d \mathbf{R} d \mathbf{r}_{\mathbf{3}} \ldots d \mathbf{r}_{\mathbf{N}}
$$

correspond to the original, total Hamiltonian and to $H_{m i x}$ respectively. They are the fully quantum mechanical free energies.

Introducing the binary radial distribution function, from the definition Eq. A1 applied to a two particle system,

$$
g_{b i n}(r)=\left(4 \pi \hbar^{2} \beta / m\right)^{2}\left\langle\mathbf{r}\left|e^{-\beta H_{r e l}}\right| \mathbf{r}\right\rangle
$$

the expansion Eq. A10 for $g(r)$ becomes

$$
g(r)=g_{b i n}(r) \Omega\left(\frac{m}{4 \pi \hbar^{2} \beta}\right)^{3 / 2} e^{-\beta\left(F_{\text {mix }}-F_{\text {pure }}\right)} \frac{\left\langle\mathbf{r}\left|e^{-\beta\left(H_{\text {rel }}+C r_{12}^{2}\right)}\right| \mathbf{r}\right\rangle}{\left\langle\mathbf{r}\left|e^{-\beta H_{r e l}}\right| \mathbf{r}\right\rangle}+O\left(\left\langle r^{4}\right\rangle\right) .
$$

The term following $g_{b i n}$ cancels the remaining "ideal gas term" in the difference between the $N$ particle $F_{\text {pure }}$ and the $N-1$ particle $F_{m i x}$. In the following this term is omitted and the free energies refer only to the nonideal terms.

For the classical case $H_{r e l}$ and $C r^{2}$ commute and the last ratio in equation above is just $e^{-C r^{2}}$ so

$$
g(r)=g_{b i n}(r) e^{-\beta\left(F_{m i x}-F_{\text {pure }}-C r^{2}\right)}=g_{b i n}(r) e^{-\beta\left(F_{m i x}-F_{\text {pure }}\right)-\frac{\Gamma}{4}(r / a)^{2}} .
$$

This relation, with the correct $r^{2}$ term, was first derived in reference 16 correcting an earlier factor of two error in reference 2 .

As a simple application consider the weak coupling, Debye-Hückel limit. Using the well known interaction free energy for this mode ${ }^{18}$

$$
F=-\frac{2}{3} \sqrt{\frac{\pi}{T V}}\left(\sum_{s} N_{s} Z_{s}^{2}\right)^{3 / 2}
$$

applied to the "pure" $\left(N_{1}=N, Z_{1}=Z\right)$ and the "mixture" case $\left(N_{1}=N-2, Z_{1}=Z, N_{2}=1, Z_{2}=2 Z\right)$ gives $h(0)=\ln \left[g(0) / g_{b i n}(0)\right]=\sqrt{3 \Gamma}$. This result also comes from expanding the screened potential form, $g(r)=e^{-\kappa r} / r$ with $\kappa$ the inverse Debye-Hückel screening length. The next term in the expansion, linear in $r$, is seen to be incorrect however.

In the quantum mechanical case the terms $H_{r e l}$ and $C r^{2}$ do not commute. The identity

$$
\begin{aligned}
e^{-\beta\left(H_{r e l}+C r^{2}\right)} & =e^{-\beta H_{r e l}}-\int_{0}^{\beta} e^{-(\beta-s) H_{r e l}} C r^{2} e^{-s\left(H_{r e l}+C r^{2}\right)} d s \\
= & e^{-\beta H_{r e l}}-\int_{0}^{\beta} e^{-(\beta-s) H_{r e l}} C r^{2} e^{-s H_{r e l}} d s+O\left(r^{4}\right)
\end{aligned}
$$

leads to

$$
\frac{\left\langle\mathbf{r}\left|e^{-\beta\left(H_{r e l}+C r^{2}\right)}\right| \mathbf{r}\right\rangle}{\left\langle\mathbf{r}\left|e^{-\beta H_{r e l}}\right| \mathbf{r}\right\rangle}=1-\beta C<r^{2}>+O\left(<r^{4}>\right)
$$

where

$$
\left\langle r^{2}\right\rangle \equiv \frac{1}{\beta} \int_{0}^{\beta} d s \int d \mathbf{r}^{\prime} \rho_{r e l}\left(\mathbf{r}, \mathbf{r}^{\prime} ; \beta-s\right) r^{\prime 2} \rho_{r e l}\left(\mathbf{r}^{\prime}, \mathbf{r} ; s\right) / \rho_{r e l}(\mathbf{r}, \mathbf{r} ; \beta)
$$

Unlike the classical case $\left\langle r^{2}\right\rangle \neq r^{2}$ but, because of the range of $\rho_{r e l}$, will differ from it by an amount proportional to the squared de Broglie thermal wavelength. For example, if $\rho_{r e l}$ is approximated by only the free particle term then

$$
<r^{2}>=r^{2}+\hbar^{2} \beta / M .
$$

When $r \rightarrow 0,<r^{2}>$ and higher order terms are thus nonzero and the simple, classical relation between the screening function at $r=0$ and the free energy difference no longer applies. What has been generated is a double expansion in $r^{2}$ and the squared ratio of the de Broglie thermal wavelength to the ion sphere radius. 
TABLE III: $\alpha(0, \beta)$ as defined in Eq. A22 and A18

\begin{tabular}{|c|c|}
\hline$\beta$ & $\alpha(0, \beta)$ \\
\hline 0.0 & 1.0 \\
0.5 & 1.106 \\
1.0 & 1.148 \\
2.0 & 1.206 \\
4.0 & 1.286 \\
6.0 & 1.345 \\
8.0 & 1.394 \\
10.0 & 1.435 \\
20.0 & 1.591 \\
30.0 & 1.703 \\
\hline
\end{tabular}

The lowest order quantum correction to the free energy ${ }^{20}$

$$
\beta F=\beta F_{\text {classical }}+\frac{\hbar^{2} \beta^{2}}{24}\left\langle\sum_{j} \frac{\nabla_{j}^{2} U}{M_{j}}\right\rangle
$$

where $U$ is the total potential energy function, applied to the uniform background model and following the algebra leading to equation $\mathrm{A} 9$ gives

$$
\beta\left(F_{\text {mix }}-F_{\text {pure }}\right)=\beta\left(F_{\text {mix }}^{\text {classical }}-F_{\text {pure }}^{\text {classical }}\right)-\frac{1}{8} \eta \Gamma .
$$

The quantum correction in this term would further increase the enhancement factor contrary to what is found.

The $\beta C<r^{2}>$ term in Eq. A17 corrects this. We have not found a simple expression for $<r^{2}>$ in terms of continuum Coulomb wave functions however it is not difficult to evaluate numerically using the axial symmetry of the $\mathbf{r}^{\prime}$ integral (or radial symmetry when $\mathbf{r}=0$ ). The result can be expressed as

$$
\left\langle r^{2}\right\rangle(r, \beta) \equiv r^{2}+\alpha(r, \beta) \frac{\hbar^{2} \beta}{M}
$$

where the function multiplying the free particle result, $\alpha(r, \beta) \rightarrow 1$ as $\beta \rightarrow 0$. As $\beta$ increases $\alpha$ slowly increases, reflecting the tendency of the repulsive Coulomb potential to emphasize larger radius "paths" compared to the free particle limit.

Adding this term to the free energy change, the lowest order quantum correction in the enhancement factor is

$$
\Delta h_{Q M}(0)=-\frac{\eta}{4}\left[\alpha\left(0, \Gamma^{2} / \eta\right)-1 / 2\right]
$$

This now correctly predicts the decrease in $h(0)$ due to quantum effects. $\alpha(0, \beta)$ is tabulated in table III. This semiclassical expansion was compared with the PIMC results for the case $\eta=0.1$ in Fig. 6. For values of $\eta \geq 0.25$ the lowest order expansion overestimates the quantum effects by almost a factor of 2 .

We thank H. DeWitt for numerous conversations and for several of the classical $h(0)$ values displayed in figure 3 . This work was performed under the auspices of the U.S. Department of Energy by University of California LLNL contract No. W-7405-Eng-48 in a CREM free environment.

1 E. E. Salpeter, Aust. Jour. of Phys.7, 373 (1954).

${ }^{2}$ H. E. Dewitt, H. C. Graboske and M. S. Cooper, Astrophysical J. 181439 (1973).

${ }^{3}$ H. E. Dewitt and W. Slattery, Contrib. in Plasma Phys. 39, 133 (1999). Also H. E. Dewitt private communication.

${ }^{4}$ S. Ogata, Phys. Rev. Lett. 772726 (1996).

5 S. Ogata, Astrophysical J. 481883 (1997).

6 E. L. Pollock and B. Militzer, Phys. Rev. Lett. 92 021101-1 (2004).

7 Nakoi Itoh, Fumiyoshi Kuwashima and Hiroharu Munakata Astrophysical J. 362620 (1990).

8 Donald D. Clayton, Principles of Stellar Evolution and Nucleosynthesis, Univ. of Chicago Press (1983).

${ }^{9}$ Lowell S. Brown and R. F. Sawyer, Rev. Mod. Phys. 69, 411 (1997). 
10 E. L. Pollock, Comp. Phys. Comm. 52, 49 (1988).

11 S. G. Brush, H. L. Sahlin, and E. Teller, J. Chem. Phys. 45, 2102 (1966).

12 E. L. Pollock and D. M. Ceperley, Phys. Rev. B 30, 2555 (1984).

13 D. M. Ceperley, Rev. Mod. Phys. 67, 279 (1995).

14 J.-M. Caillol and D. Gilles, J. Phys. A: Math. Gen. 366243 (2003)

15 B. Widom, Jour. of Chem. Phys. 39, 2808(1963).

16 B. Jancovici, Jour. of Stat. Phys. 17, 357 (1977).

17 A. Alastuey and B. Jancovici, Ap. J. 226, 1034 (1978).

18 L. D. Landau and E. M.Lifshitz, Statistical Physics, Pergamon Press (1969) Section 75.

19 H. E. DeWitt, private communication.

20 L. D. Landau and E. M.Lifshitz, Statistical Physics, ibid. Section 33.

21 Setsuo Ichimaru and Hikaru Kitamura, Phys. of Plasmas 6, 2649 (1999). Table VIII

22 D. M. Ceperley, J. Stat. Phys. 63, 1237 (1991).

23 M. D. Jones and D. M. Ceperley. Phys. Rev. Lett. 76, 4572 (1996).

24 Alfred A. Kugler, Ann. Phys. 53, 133 (1969).

25 J. P. Hansen and P. Viellefosse, Phys. Lett. 73A, 187 (1975).

${ }^{26}$ Guy S. Stringfellow, H. E. DeWitt and W. L. Slattery. Phys. Rev. A 41, 1105 (1990). 